DOI :https://doi.org/10.31692/ICIAGRO.2020.0181

\title{
FOOD DEFENSE: GERENCIAMENTO DE RISCOS E APLICAÇÃO DE FERRAMENTA CARVER + SHOCK EM FRIGORÍFICO
}

\section{FOOD DEFENSE: RISK MANAGEMENT AND CARVER + SHOCK TOOL APPLICATION IN SLAUGHTERHOUSE}

\author{
AGUIAR, D. R. ${ }^{1}$; KUSHIDA, M. M. ${ }^{2}$; PAIVA, L.C. ${ }^{3}$
}

\begin{abstract}
Resumo
A ferramenta CARVER + Shock é um software criado pelo governo norte-americano e direcionado para prever as possíveis vias de contaminação intencional de uma empresa de alimentos, propondo soluções a essas vulnerabilidades. Como objetivo para esse trabalho aplicou-se a ferramenta em um frigorífico com a finalidade de elencar os pontos críticos e vulneráveis da empresa e propor suas possíveis soluções. A metodologia proposta foi adaptada diretamente do software: através de questionários propostos na execução do programa são apresentadas questões sobre as linhas de produção, as estruturas prediais, os acessos às dependências e às informações da empresa e o controle de acessos dos colaboradores aos diversos setores da empresa. Ao final é apresentado um relatório com os principais pontos vulneráveis da empresa. Os resultados apresentados pelo software conseguiram determinar as áreas vulneráveis de contaminação intencional nas dependências do frigorífico. Além disso, o sistema conseguiu analisar locais da empresa onde geralmente não são pensadas ações de controle e definiu algumas medidas importantes para a mitigação das contaminações intencionais. Ao final concluiu-se que o sistema CARVER + Shock é uma ferramenta útil para pequenas e médias empresas do setor alimentar, apesar de ser trabalhoso e exigir um conhecimento avançado da língua inglesa.
\end{abstract}

Palavras-chave: Segurança dos alimentos, Food Defense, CARVER+Shock, frigorífico.

\begin{abstract}
The CARVER + Shock tool is a software created by the US government and aimed at predicting possible ways of intentional contamination by a food company, proposing solutions to these vulnerabilities. As a goal for this work, the tool was applied in a slaughterhouse with the purpose of listing the critical and vulnerable points of the company and proposing its possible solutions. The proposed methodology was adapted directly from the software: through questionnaires proposed in the execution of the program, questions are presented about the production lines, building structures, access to the company's premises and information and the control of access by employees to the various sectors of the company. At the end, a report is presented with the main vulnerable points. The results presented by the software were able to determine the vulnerable areas of intentional contamination on the premises of the slaughterhouse. In addition, the system was able to analyze company locations where control
\end{abstract}

\footnotetext{
${ }^{1}$ Mestre em Ciências e Tecnologia de Alimentos pelo Instituto Federal de Educação, Ciência e Tecnologia do Sul de Minas, campus Machado. E-mail: danielle.resende @ hotmail.com.br.

2 Doutora em Ciências de Alimentos pela Universidade Estadual de Campinas (UNICAMP) Professora/pesquisadora da Faculdade de Zootecnia e Engenharia de Alimentos da Universidade de São Paulo FZEA/USP. E-mail: martakushida@usp.br.

${ }^{3}$ Doutor em Agronomia pela Universidade Federal de Lavra (UFLA), Professor titular do Instituto Federal de Educação, Ciência e Tecnologia do Sul de Minas, campus Machado. E-mail: leandro.paiva@ifsuldeminas.edu.br.
} 
actions are not usually thought of and defined some important measures to mitigate intentional contamination. In the end, it was concluded that the CARVER + Shock system is a useful tool for small and medium sized companies in the food sector, despite being laborious and requiring an advanced knowledge of the English language.

Keywords: Food Safety, Food Defense, CARVER + Shock, slaughterhouse.

\section{Introdução}

Nas últimas décadas, a globalização, somada a exigência dos mercados, vem impor às empresas a adoção de sistemas mais consistentes e eficazes para satisfazer as necessidades atuais, no que concerne à segurança e qualidade alimentar. As crescentes preocupações das empresas, governos e consumidores sobre a contaminação intencional e fraude alimentar conduziram a emergência de novos conceitos e metodologias como o da defesa alimentar, cujo escopo visa essencialmente a proteção da indústria, produtos e instalações, da contaminação intencional, adulteração e terrorismo alimentar (SEVERINO, 2016).

Esta iniciativa exige o esforço corporativo e governamental para combater os acontecimentos de adulterações, os quais devem estar cientes das ameaças terroristas a que o país pode estar sujeito e do impacto que poderá ter sobre a cadeia alimentar. Para ir ao encontro a estes esforços, as empresas do setor alimentar devem dispor de meios e procedimentos para prevenir e antecipar situações de contaminação intencional ou adulteração. Segundo a Organização Mundial de Saúde (OMS), o combate ao terrorismo alimentar é conseguido utilizando-se de medidas articuladas de prevenção, detecção, preparação, resposta e comunicação entre os países, instituições e pessoas. Para que tais medidas sejam concretizadas com sucesso é crucial que os países tenham previamente implantados programas de segurança e defesa alimentar consistentes e eficazes (WHO, 2002).

Nesse sentido, o food defense (em tradução livre, defesa alimentar) é um conceito que tem surgido com objetivo de desenvolver uma abordagem de programas visando proteger, prevenir e mensurar o impacto sobre a população e a indústria produtora de alimentos passíveis de um ataque de contaminação intencional. A segurança dos alimentos (food safety) encontrase associada à garantia do fornecimento de produtos seguros ao longo de toda a cadeia alimentar, garantindo que um alimento não causará prejuízos à saúde do consumidor por contaminações biológicas, químicas ou físicas, quando é preparado e/ou no momento que é ingerido, segundo a utilização prevista (BETANCOURT, 2017). A segurança alimentar (food security) na perspectiva de garantia da disponibilidade dos alimentos como meio de garantir a subsistência das populações, é definida segundo a Food and Agriculture Organization on th 
United Nations (FAO) como a garantia para todos os seres humanos do acesso físico e econômico, estabilidade dos alimentos e utilização de alimentos seguros e saudáveis, sendo assim, uma questão de envolvimento governamental, através de políticas públicas (FAO, 1996).

Ao trazer a temática à nossa realidade, o Brasil apresenta uma agricultura muito desenvolvida devido à suas dimensões continentais e a quantidade de alimento exportado a todos os países do mundo. O Ministério da Agricultura, Pecuária e Abastecimento (MAPA), criado há mais de 150 anos, é responsável pela garantia da segurança alimentar e Segurança dos Alimentos, juntamente com a Agencia Nacional de Vigilância Sanitária (ANVISA), esta representante do Brasil no CAC - Codex Alimentarius Commision, este último criado pela FAO (Organização das Nações Unidas para a Agricultura e Alimentação). Em 1963, o CAC lançou o Codex Alimentarius, um manual de procedimentos que visa à inocuidade dos alimentos. A partir disto, foram exigidas das empresas que processam, embalem e distribuem alimentos o cumprimento de requisitos mínimos de qualidade. Nesse momento, qualidade em alimento é obrigatório a toda a indústria alimentícia, independente do poder aquisitivo ou nível de exigência do consumidor (BETANCOURT, 2017).

Com o mercado mundial de exportação e importação cada vez desenvolvido, em 1993, o sistema Analises de Perigos e Pontos Críticos de Controle (APPCC) foi incorporado ao Codex Alimentarius e exigido a todos os países membros do CAC. Este sistema visa prevenir as contaminações intencionais ou não intencionais dentro da linha de processamento, com o objetivo de analisar os perigos e os pontos críticos de controle. Em 1998, o MAPA adotou o APPCC como ferramenta que aprimora o sistema de inspeção dentro das indústrias de processamento de alimento no Brasil. Este é o primeiro sistema de qualidade que muda o conceito de controle de qualidade para garantia de qualidade. Atualmente é o principal método em operação em nosso país que garante a qualidade na produção de alimentos (GERMANO; GERMANO, 2015).

A partir de 2001, principalmente, após os eventos terroristas de 11 de setembro, houve uma maior preocupação com o tratamento da contaminação intencional como possibilidade de prejuízo à garantia de qualidade. Com os atentados, nos Estados Unidos, a U.S. Food and Drug Administration (FDA) passou a intensificar os trabalhos na modernização do sistema de qualidade, com o objetivo de extrapolar o APPCC para além da linha de produção de alimentos, analisando todos os pontos vulneráveis da empresa. A partir dessas observações iniciou-se uma discussão sobre um alimento defendido ou protegido de ameaças sob a expressão Food Defense (FDA, 2014). 
Baseando-se nesses fatos, estabeleceu-se uma política para identificar infraestruturas críticas, incluindo sistemas de produção de alimentos e agricultura, com intuito de protegê-los de ataques terroristas. A criticidade foi definida como o impacto negativo que um ataque ou falha de uma dada infraestrutura teria na nação se ela fosse comprometida ou destruída (SAATHOFF, 2011).

De acordo com Manning \& Soon (2016), o conceito de food defense pode ser descrito como o processo que garante a segurança dos alimentos, bebidas e das suas cadeias de abastecimento de todas as formas de adulteração intencional. De um modo geral, estes atos são motivados por fins econômicos ou com o objetivo de causar danos à saúde pública.

Um plano de defesa alimentar (food defense) é basicamente um sistema que visa proteger os produtos alimentares de adulteração intencional. $\mathrm{O}$ desenvolvimento de um plano garante um ambiente de trabalho seguro para os funcionários, fornece um produto seguro, protege o negócio da empresa e aumenta o grau de preparação e a capacidade de resposta perante uma situação crítica (SEVERINO, 2016).

Existem diversos sistemas de defesa alimentar cujo escopo visa a prevenção da contaminação intencional de produtos, no qual indivíduos, motivados ideologicamente ou não, estão propensos a contaminar produtos com recurso a determinados agentes, com a finalidade de causar prejuízos diretos às organizações ou à população em geral. Os sistemas de defesa alimentar garantem ainda a proteção contra outras formas de agressão à empresa, como danos à propriedade, à marca ou aos funcionários da organização (HUFF, 2014).

Atualmente não existe nenhuma estrutura específica definida para um plano de defesa alimentar, este deve ser desenvolvido considerando vários fatores, que podem incluir o ambiente e envolvente da unidade, a respectiva construção e segurança (interna, externa, pessoal) e a acessibilidade às instalações. $\mathrm{O}$ desenvolvimento de um plano de defesa alimentar exige uma análise prévia pela organização da necessidade de implementação do mesmo, avaliação dos recursos disponíveis e assegurar o envolvimento de uma equipa nesta área (CTCPA, 2012).

Com base nos sistemas existentes para a defesa alimentar, a empresa pode desenvolver um plano baseado nas ferramentas auxiliares. Estes sistemas permitem a implementação e integração nos planos de segurança dos alimentos, aspectos que cobrem a avaliação de ameaças, de vulnerabilidades ou de riscos com vista à prevenção de contaminação intencional, adulteração e fraude alimentar (aspetos cobertos pela defesa alimentar).

Para auxiliar a indústria de alimentos norte-americana na produção de seus planos de defesa alimentar, a FDA, em trabalho conjunto com o United States Department of Agriculture 
(USDA), desenvolveu uma ferramenta tecnológica para evitar contaminações deliberadas, denominado CARVER + Shock. O CARVER é um acrônimo que relaciona seis atributos usados para avaliar a atratividade de um alvo para o ataque, dos quais nomeiam-se (FDA, 2014):

- Criticidade: impacto na saúde pública e economia resultante de um ataque. A introdução de agentes de ameaça nos alimentos pode causar impactos significativos na saúde pública ou economia;

- Acessibilidade: capacidade de acesso físico e saída do invasor. Uma organização é acessível quando um invasor pode alcançar a área ou etapa do processo e realizar o ataque sem ser identificado. Esta medida é independente da probabilidade de sucesso da introdução de agentes de ameaça;

- Recuperabilidade: capacidade de recuperação do sistema após um ataque. Este critério é medido com base no tempo que o sistema específico leva a recuperar a produtividade, considerando a diminuição do volume de vendas, associado à baixa procura do produto;

- Vulnerabilidade: facilidade de realizar ataque. Medida da facilidade com que os agentes de ameaça podem ser introduzidos em quantidades suficientes para atingir o propósito do invasor. A vulnerabilidade é determinada tanto pelas caraterísticas do alvo e como as do meio ambiente circundante;

- Efetividade: quantidade de perdas diretas na produção resultante do ataque. Este critério mede a perda de produtividade do sistema danificado por um ataque num único setor da instalação. Portanto, para o mesmo produto, o efeito é inversamente relacionado com o número total de instalações;

- Reconhecimento: facilidade de identificação de alvo. Medida de reconhecimento de um invasor, sem confundir com outros alvos ou componentes.

O critério Shock constitui o sétimo atributo considerado na metodologia CARVER. É a medida combinada dos impactos econômicos, de saúde e psicológicos resultantes de um ataque bem-sucedido. O impacto psicológico será aumentado se houver um grande número de mortes ou caso o alvo tenha um significado simbólico, histórico, cultural, religioso ou outro. As repercussões na economia são caraterizadas pela diminuição da atividade econômica nacional, o aumento do desemprego nas indústrias em causa, entre outros fatores inerentes (FDA, 2014). 
O software avalia as vulnerabilidades dentro de um sistema ou uma edificação. Facilita ao usuário pensar como o invasor, identificando os alvos mais atraentes ou mais propensos para um ataque, o que permite concentrar os recursos e planos de proteção nestes pontos. $\mathrm{O}$ CARVER + Shock produz uma série de questionários criados para avaliar os possíveis pontos de vulnerabilidade das empresas e sugerir possíveis mudanças de comportamento por parte dos gestores. O CARVER + Shock também é responsável por criar um plano de recuperabilidade da empresa, caso aconteça uma ameaça. Esta é uma ferramenta não utilizada em solo brasileiro no que tange a indústria de alimentos.

Sendo assim, o principal objeto deste artigo, como divulgação dos resultados de dissertação de mestrado, foi trazer o conhecimento e uma análise dessa ferramenta e aplicá-la em uma empresa do setor alimentício, buscando analisar as vulnerabilidades da empresa como um todo. A empresa escolhida foi um frigorífico de abate de suínos que atua no Estado de São Paulo. O trabalho teve como hipótese o fato que esse software poderia ser utilizado em adição ao trabalho já desenvolvido pela equipe de implantação do sistema APPCC. Como a ferramenta prevê ao usuário uma visão como um terrorista ao aplicar uma contaminação intencional, o software poderia atuar em locais onde o sistema APPCC não teria o foco, prevendo as vulnerabilidades da empresa. A partir dos resultados apresentados pelo CARVER + Shock é possível, então, conseguir desenhar e implementar planos de defesa alimentar para a empresa. Estes planos poderiam minimizar o risco de contaminação intencional e obter uma resposta rápida em caso de uma emergência que seja consequência de uma contaminação.

\section{Materiais e métodos}

\section{Descrição da empresa analisada}

A empresa, objeto do estudo, doravante denominada empresa $X$, é uma empresa de capital nacional, localizada no Estado de São Paulo e atua no ramo de abate de suínos desde 1978. A empresa possui um frigorífico de porte médio, contando com 217 colaboradores, sendo que 80 trabalham diretamente com a linha de abate de suínos e uma desossa, gerando uma produção de 800 toneladas por mês de produtos cárneos, fazendo parte deste portfólio, cortes cárneos como: carne resfriada com osso (meia carcaça suína), sendo este o principal produto que é revendido para a maior parte dos açougues de supermercado de grandes redes e para açougues convencionais; carne congelada sem osso; carne congelada com osso e miúdos de suínos resfriados e congelados. O frigorífico tem autorização, expedida pelo Ministério da 
Agricultura, Pecuária e Abastecimento (MAPA) para o abate de 1000 animais por dia, e entrepostagem de 400 quartos de carcaça de bovinos. Além disso, a empresa possui o APPCC implantado e está em fase de implantação de ISO 9001. Neste momento, um novo setor está em fase final de implantação (setor de fabricação de embutidos), que pretende processar até 5 toneladas diárias, aumentando, assim, sua cadeia de atuação dentro do setor de carnes, fechando, praticamente, toda a cadeia de produção.

Um dos motivos pelo qual visualizou-se a necessidade de estudar a ferramenta CARVER + Shock e implantá-la na empresa em questão deveu-se ao incidente de uma contaminação intencional por creolina praticada por um funcionário insatisfeito com sua chefia. Na ocasião, o funcionário responsável pela desinfecção das pocilgas de recebimento de suínos tece acesso a uma quantidade maior de creolina do que a descrita nos Procedimentos Operacionais Padronizados (POP's), no próprio almoxarifado de produtos químicos. O funcionário, descontente com a atuação de seu superior imediato, cumpriu todo o procedimento de desinfecção das pocilgas, sendo o mesmo verificado pelos colaboradores responsáveis pela garantia da qualidade. Logo depois da desinfecção, com a creolina restante (cerca de 5 litros), o funcionário, próximo do término de seu turno de trabalho, se dirigiu até o reservatório de água potável do estabelecimento e depositou a creolina diretamente no reservatório. $\mathrm{O}$ ato foi cometido numa segunda-feira. No outro dia, durante a vistoria pré-operacional realizada pelo setor de garantia de qualidade, foi constatada a contaminação do reservatório de água, observada nas análises de água, no qual constatou-se alterações de $\mathrm{pH}$, odor e turbidez. Constatadas as alterações, o abate foi suspenso pela equipe da garantia da qualidade e pela equipe da Inspeção Federal do MAPA. O fiscal local acionou a fiscalização regional que aplicou um primeiro auto de infração (multa) por falta de água potável para o abate. Um segundo auto de infração foi aplicado à empresa, por conta de presença de cresóis em níveis não aceitáveis no reservatório de água; essa amostra foi colhida no dia seguinte e enviada ao LANAGRO (Laboratório Nacional Agropecuário) vinculado ao MAPA.

Os prejuízos com a interdição do abate foram: 2 mil carcaças que deixaram de ser vendidas, totalizando 160 toneladas de carne que deixaram de ser produzidas; gastos extras com alimentação, água e limpeza; gastos extras com a dispensa e pagamento de funcionários pelos 4 dias de interdição; pagamento das multas acarretadas pelos autos de infração; o histórico negativo por água contaminada no processo adquirida pelo estabelecimento dentro do Ministério da Agricultura. O caso não foi noticiado pelos meios de comunicação, sendo assim o estabelecimento não sofreu deterioração de sua imagem junto ao consumidor final, mas o cliente intermediário não foi atendido prontamente com a mercadoria. 
Percebeu-se, assim, a necessidade de procurar ferramentas que pudessem cobrir toda a empresa, para além do APPCC, que já era aplicado, mas visava apenas as linhas de produção e anexos, mas não a empresa como um todo. A empresa necessitava de um sistema que sistematizasse a autorização e os níveis de acesso de cada colaborador em setores mais vulneráveis a ataques. Pensando dessa forma, a pesquisa foi proposta com a implantação do sistema CARVER + Shock nos setores da empresa considerados vulneráveis e sua avaliação quanto à sugestão de mitigação dessas vulnerabilidades e acessibilidade a esses setores.

\section{Descrição do software CARVER + Shock}

O software CARVER + Shock foi desenvolvido, inicialmente, para analisar e mitigar as vulnerabilidades apresentadas nas instalações do exército dos Estados Unidos. Após os incidentes terroristas de setembro de 2001, a agência americana de vigilância sanitária (FDA), juntamente com o setor de agricultura (USDA) adaptaram o software para as necessidades das empresas produtoras de alimentos dos Estados Unidos (FDA, 2015).

O software possui dois segmentos principais: um primeiro direcionado aos produtores agrícolas, no qual, um questionário, bem como os resultados, são voltados às vulnerabilidades concernentes à produção agrícola e aos agentes que possam perpetrar algum ato terrorista nas instalações. Um segundo segmento é direcionado às empresas de processamento de alimentos; as entradas de dados são direcionadas para as estruturas e os fluxogramas de produção na qual as empresas são especializadas. Tanto as perguntas, quanto as sugestões apresentadas, tem o objetivo de mitigar as vulnerabilidades do setor produtivo, bem como do entorno da empresa.

O software contém três seções principais: fluxograma, entrevista e pontuação. Inicialmente, a primeira página do programa sugere que o usuário insira os dados, elaborando uma representação gráfica do processo de produção em forma de fluxograma. Esta seção possui a alternativa de agregar ícones que representam as operações realizadas, os processos de produção, os ingredientes e materiais empregados para que se possa realizar toda a produção da empresa analisada (FDA, 2014). O fluxograma deve ser elaborado contemplando todas as etapas do processo de produção: desde a recepção da matéria prima, acondicionamento, processo produtivo, descarte de subprodutos, armazenamento e transporte de produtos acabados, bem como o transporte dos subprodutos e dos rejeitos produzidos no processo de fabricação do produto. A Figura 1 mostra o fluxograma da empresa X analisada. Nele pode-se perceber como o processo produtivo do abate de suínos foi montado, mostrando ao leitor todas as etapas do processo: 
Figura 1 - Apresentação do fluxograma desenvolvido no programa CARVER + Shock.

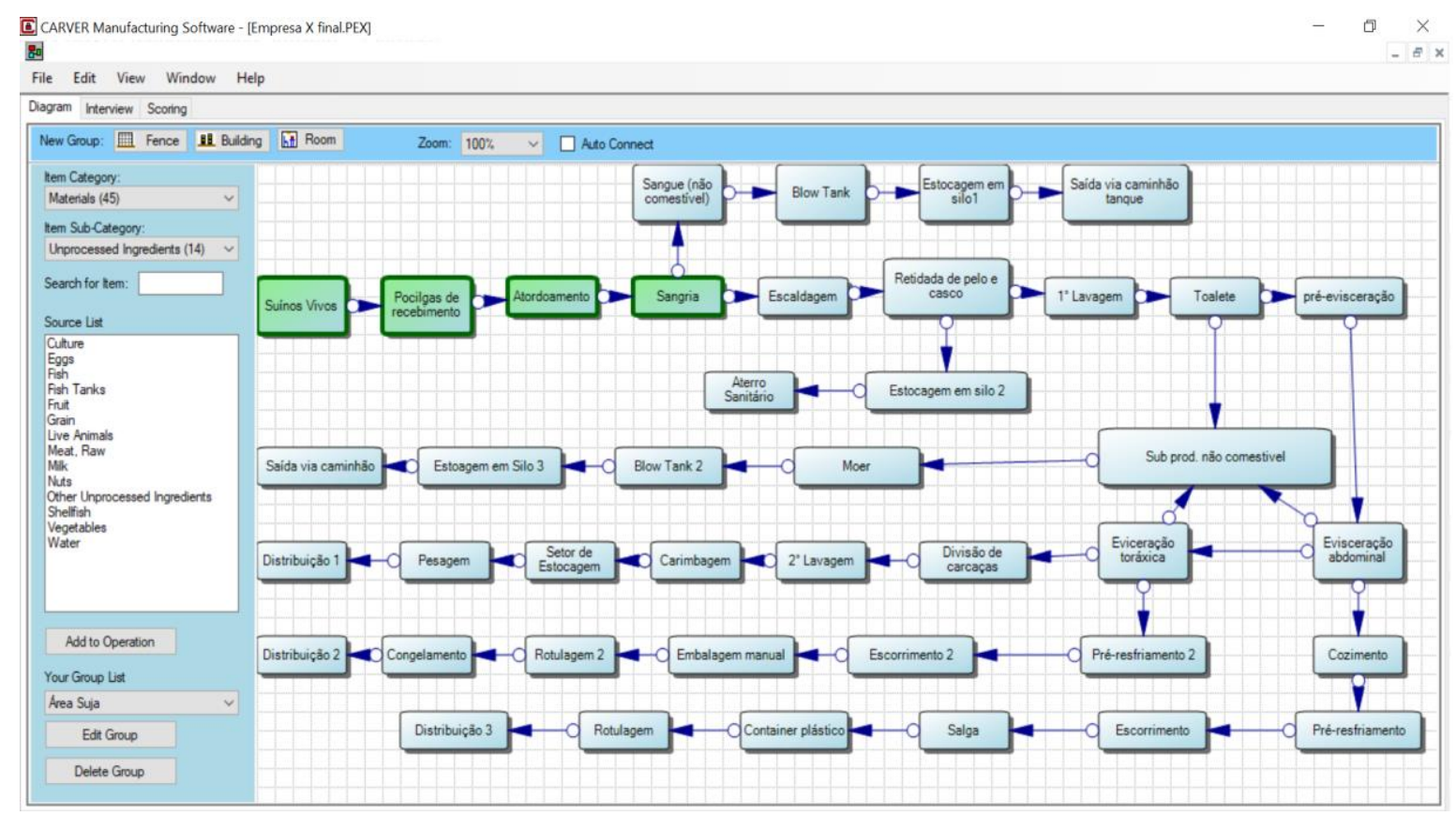

Fonte: CARVER+Shock Industry

Logo após a construção do fluxograma de processos o software gera um questionário contendo várias perguntas. As perguntas são específicas para cada ícone criado anteriormente no fluxograma. Elas são baseadas em pontos que podem ser suscetíveis ao acesso por funcionários, visitantes, funcionários terceirizados, motoristas, funcionários de empresas de suporte e manutenção, além de vendedores externos.

Um modelo pode ser descrito na Figura 2 abaixo, no qual pode-se observar, no canto esquerdo, cada setor criado pelo fluxograma. Se o usuário clicar em cada setor, será colocado à disposição uma série de perguntas concernentes àquele setor. Além disso, serão providenciadas perguntas que envolvam a segurança da empresa como um todo, bem como o tratamento dado a visitantes e membros externos à empresa. Na parte inferior estão todas perguntas que precisam ser respondidas: 
Figura 2 - Questionário gerado a partir do fluxograma para o item Facility.

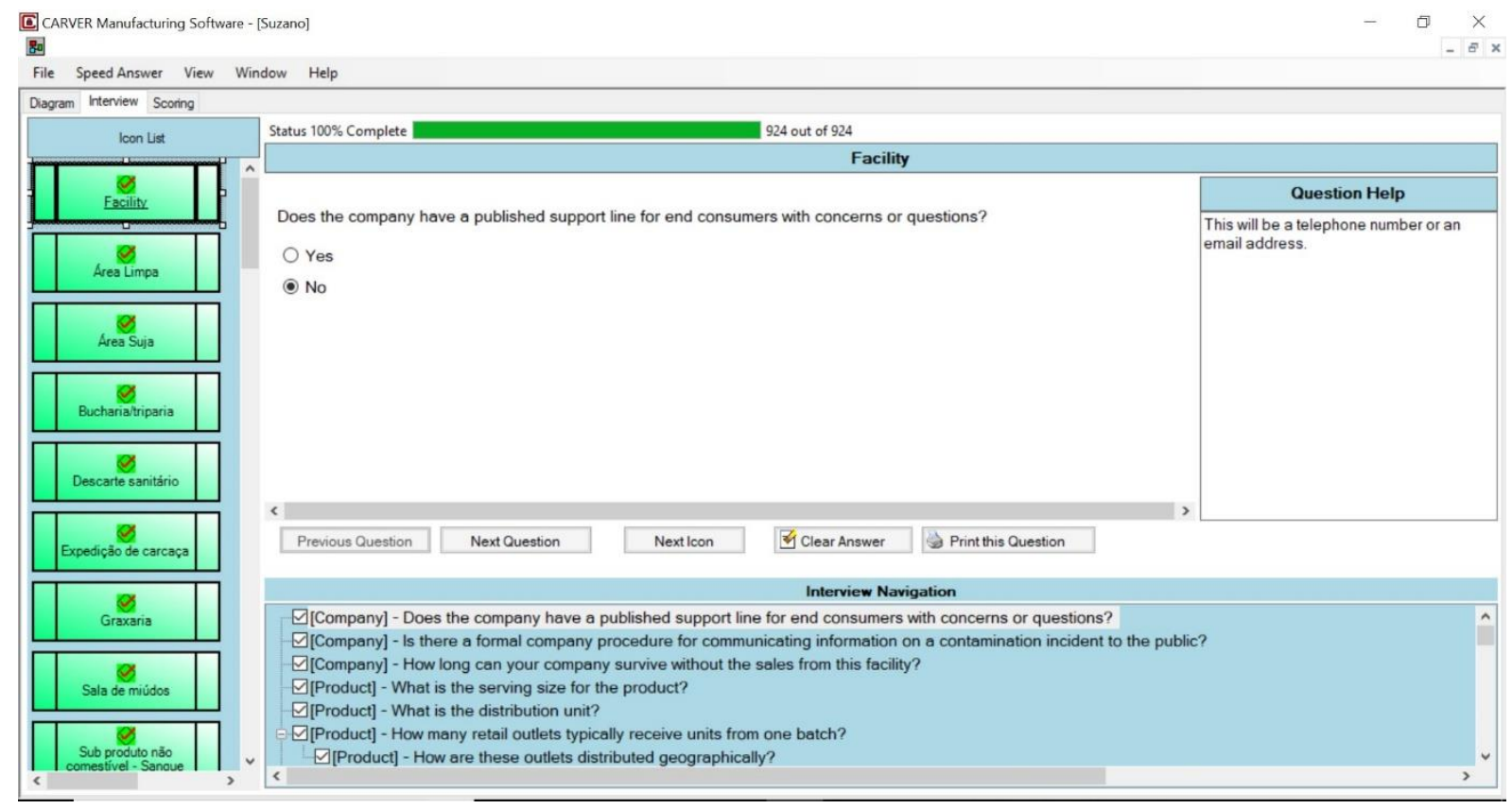

Fonte: CARVER + Shock Industry.

Após todas as perguntas propostas serem respondidas - no caso da pesquisa foram quase 950 perguntas - cada item foi qualificado e avaliado com base nos sete atributos (descritos anteriormente) que compõem o sistema CARVER + Shock, apresentando uma pontuação geral do sistema. Os atributos foram avaliados numa escala de 1 a 10 , no qual quanto mais elevada foi a pontuação, maior a vulnerabilidade, e portanto, são considerados pontos mais suscetíveis a algum ataque (FDA, 2014).

Para avaliar os resultados o software utilizou dois gráficos em forma de barras em uma escala de 0 a 20, cada um com uma cor e um grupo de atributos específicos. A barra de cor azul é denominada "importância" e representa os atributos de criticidade e recuperabilidade. A barra de cor vermelha é chamada de "facilidade de um ataque" e representa os atributos de acessibilidade e a vulnerabilidade. Para cada ícone proposto no fluxograma é apresentado o conjunto de barras que representam os atributos para cada ícone (BETANCOURT, 2017).

Além disso, no canto direito, o usuário pode perceber que para cada atributo foram oferecidas sugestões para mitigar as vulnerabilidades. Essas sugestões foram propostas pelo próprio software que analisou o grau de relevância das questões respondidas pelo usuário no 
momento da entrevista. Estes dados permitiram identificar a posição dos itens dentro dos quadros de resultados estabelecidos anteriormente pela agência de vigilância sanitária americana (FDA, 2015b). O conjunto de atributos pode ser analisado na Figura 3:

Figura 3 - Quadro de atributos das vulnerabilidades apresentadas pelo sistema.

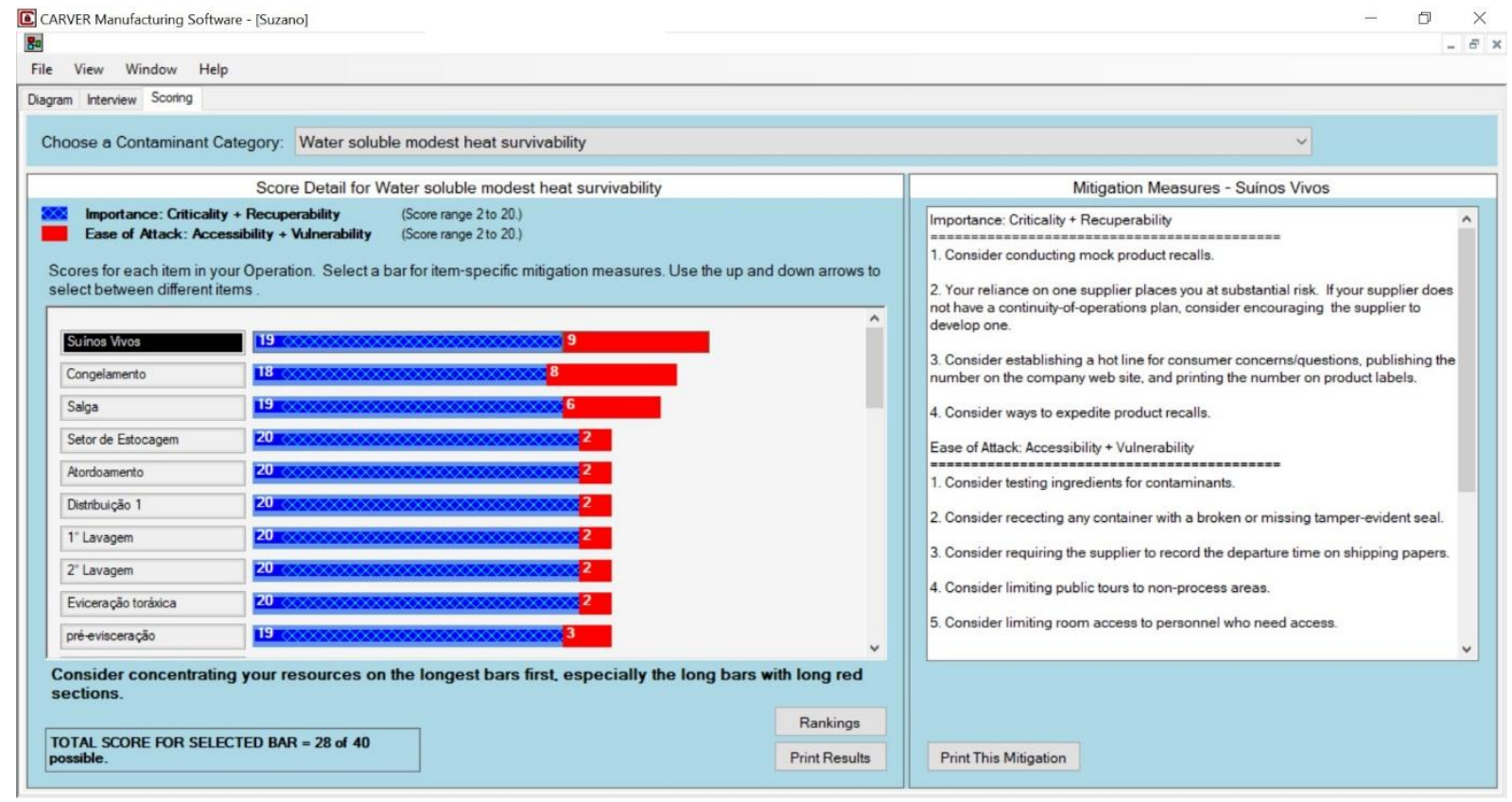

Fonte: CARVER + Shock Industry

\section{Resultados e discussão}

\section{Análise sobre o software}

Como já dito anteriormente, o software CARVER + Shock foi desenvolvido para atender as necessidades das empresas norte-americanas no desenvolvimento de planos de controle para a defesa alimentar. O objetivo, ao usá-lo como metodologia de pesquisa, foi testá-lo como ferramenta, adaptando a realidade do software ao cotidiano de uma empresa brasileira de produção de alimentos.

O primeiro dos obstáculos foi, efetivamente, o uso do idioma. Todo o software é apresentado na língua inglesa. Diante disso, o primeiro grande desafio foi montar o fluxograma e entender quais os atributos já relacionados dentro do software poderiam ser adaptáveis à realidade das empresas brasileiras, que são distintas das norte-americanas. Trata-se de abrir todas as opções de fluxogramas de cadeias de produção já existentes dentro do software e buscar 
uma que melhor adaptasse à cadeia de produção já estabelecida pela empresa $\mathrm{X}$, objeto desta pesquisa. A cadeia de produção que melhor se adaptava ao contexto da empresa $\mathrm{X}$ foi a slaughterhouse (ou abatedouro, em livre tradução). Nesta opção, o fluxograma continha etapas de abate de aves, de bovinos, peixes e suínos. As particularidades de cada fluxo, se não observadas ou não entendidas, podem levar a uma resultado não aplicável.

Outro fato, relacionado ao uso da língua inglesa, é a implantação em empresas brasileiras, sobretudo as de pequeno porte. É notório a dificuldade e o acesso da população brasileira ao ensino de uma língua estrangeira. Para empresas de pequeno porte este pode ser um limitador para a implantação. E a não implantação em pequenas empresas é um agravante, pois parte da matéria prima da maioria da cadeia de produção de alimentos brasileira, advém destes fornecedores.

Em um segundo momento, talvez o mais trabalhoso, foi a tradução de todas as perguntas propostas pelo software. Foram mais de 950 perguntas (repetitivas e quando se aplica a uma entrevista, o software fica cansativo) que davam conta de todos os processos apresentados na montagem do fluxograma dentro do software. Esses processos puderam ser melhor visualizados na Figura 1, apresentada anteriormente. Mais uma vez, a barreira da língua inglesa se apresenta como o grande entrave para o perfeito entendimento das perguntas. $\mathrm{O}$ tempo gasto na tradução, reflexão e resposta às perguntas propostas é grande e dispendioso. Além disso, no que tange as perguntas, elas não são tratadas em profundidade dentro de cada setor; são perguntas generalizadas que abordam todos os setores em conjunto.

Há um banco de dados contendo os fluxos de produção dos principais ramos da indústria de alimentos (laticínios, frigorificos etc). Dentro destes fluxos de produção, no software, as áreas são separadas em internas e externas. Diante disso, percebeu-se que poderia-se aplicar a língua portuguesa para nomear cada etapa do fluxograma, dentro dessa separação. Isto pode facilitar a visualização das áreas estudadas.

Depois do fluxograma realizado as perguntas são mostradas, baseando-se nas premissas de avaliação do CARVER + Shock, sendo levado avaliar sobre cada etapa na linha de abate ou setor/sala destinada a etapa do processo. Dado o número enorme de perguntas postuladas, evidentemente, as respostas e propostas apresentadas pelo software ao analisar os dados coletados também são enormes. Todos os resultados são apresentados ou em forma de score, contendo uma pontuação que varia de acordo com os atributos da metodologia CARVER, ou em forma de respostas curtas. Além disso, são apresentadas algumas sugestões, também baseado na mesma metodologia. Para um primeiro contato com o food defense, em uma primeira avaliação da empresa, a sistemática da lógica de "invasor", dado pelo programa, tanto 
no momento das perguntas, como nas propostas e no score apresentado ao fim da análise é interessante e um ponto positivo para o software.

Como visto nas figura 3, os dados são apresentados de maneira bastante dinâmica pelo programa através de gráficos e sugestões específicas para cada atributo selecionado. Este é outro ponto positivo para o software: ele é didático e as informações e sugestões são claras e levam ao usuário uma navegação direta sobre as decisões que ele precisa tomar para mitigar cada um dos problemas apresentados pelo software.

Ainda na figura 3, pode-se perceber uma aba superior onde mostra os principais tipos de contaminação que o programa propõe que poderiam ser perpetradas por um agente malicioso. $\mathrm{O}$ usuário pode navegar por essa aba e perceber todas as sugestões para cada tipo de contaminação diferente. Evidentemente, a navegação se torna mais complexa quanto mais informações o usuário deseja coletar. Por outro lado, há muitas sugestões e análises que se repetem nas diferentes abas do software.

\section{Conclusão}

O conceito de defesa alimentar vem assumindo um papel cada vez mais importante nos programas tradicionais de segurança dos alimentos. Os ataques ocorridos a nível mundial têm conduzido a novas exigências de comercialização e uma conscientização da necessidade de programar tecnologias para elaborar um plano de defesa alimentar contra tais ameaças, passíveis de ocorrer em qualquer ponto da cadeia alimentar. Cabe salientar que, existe apenas um trabalho anterior a este no Brasil. Trata-se da aplicação e avaliação do CARVER + Shock em dois laticínios sendo um de pequeno porte e outro de médio porte (BETANCOURT, 2017).

O sistema CARVER + Shock apresentou-se como uma ferramenta útil para pequenas e médias empresas, já que o software foi testado em um frigorífico de médio porte, com os resultados aqui publicados, e, também, com os resultados obtidos pela aplicação da ferramenta em laticínios de pequeno e médio porte obtendo resultados satisfatórios segundo BETANCOURT (2017). O fator mais preponderante que possa inviabilizar a implantação do software ainda é o uso da língua inglesa e a interpretação das informações contidas nos resultados.

No que tange a empresa analisada, o software conseguiu determinar as áreas vulneráveis de contaminação intencional nas dependências do frigorífico. O sistema conseguiu analisar locais da empresa onde geralmente não são pensadas ações de controle e definiu algumas medidas importantes para a mitigação das contaminações intencionais. 
A partir dos resultados obtidos pelo CARVER + Shock, ressalta-se que os dados coletados foram analisados e compilados. O desenvolvimento e conceito de food defense foi apresentado à equipe da garantia da qualidade, juntamente à diretoria da empresa. Foi apresentada a necessidade do desenvolvimento de um plano de defesa alimentar que seja complementar aos outros planos já implantados. Sendo assim, houve uma movimentação positiva no sentido de criar uma atmosfera segura que contemplasse tanto a produção quanto as áreas adjacentes ao frigorífico, visto que, a empresa já tinha o histórico de contaminação intencional perpetrada por funcionários.

No entanto, ainda é necessário efetuar uma avaliação de vulnerabilidade detalhada considerando vários fatores que permitam uma análise sobre o impacto econômico, psicológico e na saúde da população e dos clientes consumidores dos produtos comercializados pela empresa, depois de uma contaminação intencional.

Ao final deste estudo conclui-se que o sistema CARVER + Shock é uma ferramenta útil para pequenas e médias empresas do setor alimentar, apesar de ser trabalhoso e exigir do usuário um conhecimento avançado da língua inglesa, dado o número alto de perguntas a serem respondidas. Às empresas que adotarem esse sistema, cabem a elas construir um plano de resposta rápido caso uma contaminação ocorra, envolvendo sistemas de recall, criação de uma equipe de segurança e na divulgação efetiva de notícia, evitando a deterioração da imagem da empresa junto ao mercado consumidor.

Sendo assim, em países como o Brasil, onde a indústria agroalimentar constitui uma parte vital para a economia do país, gerando divisas quando os produtos são exportados, ou para o consumo interno, a questão da defesa alimentar assume uma extrema importância econômica; portanto é importante garantir que os produtos sejam de uma fonte confiável e cumpram com os novos requisitos normativos produzidos em outros países. É necessário estruturar planos de defesa alimentar nas empresas produtoras de alimentos em nosso país. Esses planos implantados poderão, se implantados, monitorados e verificados, baseado em ciência e tecnologia, e fundamentado nas recomendações feitas por sistemas complementares, como o CARVER + Shock, melhorar a segurança física, pessoal, operacional e assim minimizar o acesso do agressor aos processos produtivos das empresas. 


\section{Referências}

BETANCOURT, Sarah Romero. Defesa alimentar (food defense): avaliação e aplicação da ferramenta CARVER + Shock na indústria do leite no Brasil. Dissertação de mestrado. Universidade de São Paulo, 2017.

BOSONA, T; GEBRESENBET, G. Food traceability as na integral part of logistics management in food and agricultural suply chain. Food Control, vol. 33, n 1, pp. 32-48, 2013.

BRASIL. MINISTÉRIO DA AGRICULTURA, PECUÁRIA E ABASTECIMENTO. Decreto $n^{0}$ 9.013, de 29 de março de 2017. Dispõe sobre o regulamento da inspeção industrial e sanitária de produtos de origem animal, que disciplina a fiscalização e a inspeção industrial e sanitária de produtos de origem animal, instituídas pela Lei $\mathrm{n}^{\circ} 1.283$, de 18 de dezembro de 1950, e pela Lei no 7.889, de novembro de 1989. Diário Oficial da União, 29 de março de 2017.

BRASIL. MINISTÉRIO DA AGRICULTURA, PECUÁRIA E ABASTECIMENTO. Circular $n^{0}$ 175, de 16 de maio de 2005. Dispõe sobre os procedimentos de verificação dos programas de autocontrole (versão preliminar). Diário Oficial da União, 16 de maio de 2005.

BRITISH STANDARDS INSTITUTION (BSI). PAS 96: Guide to protecting and defending food and drink from deliberate attack. Londres: BSI, 2014.

BRUEMMER, Barbara. Food biosecurity. Journal of American Dietetic Association, v. 106, nº 06, june 2003, pp. 687-691.

CENTRE TECHINIQUE AGRALIMENTARE (CTCPA). La food defense: protection de la chaîne alimentaire contre les risques d'actions malvelillantes, criminelles ou terroristes. Paris, France, 02 julliet 2012. Disponível em: https://www.ctcpa.org/food-defense-securitealimentaire-ctcpa. Acesso em: 13 de junho de 2018.

DIAS, Juliane; UBARANA, Fernando. Food defence: proteção contra a contaminação intencional. Revista Controle de Contaminação. n. 147, p. 33-36, julho de 2011.

FAO. World Food Summit. (1996). Disponível em: http://www.fao.org/wfs/index_en.htm. Acesso em: 13 de junho de 2018.

FREDRICKSON, N. R. Food security: food defense and biosecurity. In: ALFEN, N. K. V. (Ed.). Encyclopedia of agriculture and food systems. Oxford: Academic Press, 2014. P. 311323.

GERMANO, Pedro M.L; GERMANO, Maria Izabel S. Higiene e vigilância sanitária de alimentos. $3^{\text {a }}$ ed. Barueri, SP: Ed. Manole, 2008.

GLOBAL FOOD SAFETY INITIATIVE FOUNDATION \& THE CONSUMER GOODS FORUM (GFSI \& CGF). GFSI Position on Mitigating the Public Health Risk of Food Fraud. France: GFSI, 2014.

HAVAS, Karyn; SALMAN, Mo. Food security: its components and challenges. Nutrition and Public Health Journal, vol. 4, $\mathrm{n}^{\circ}$ 1, 2011. 
HUFF, Andrew G. Enhacing food defense: risk managers' perceptions, critically assessments, and a novel method for objectively determining food systems' criticality. Doctor degree: University of Minnesota, USA. March, 2014.

KEREMIDIS, et. ali. Historical perspective on agoterrorism: lessons learned from 1945 to 2012. Biosecurity and bioterrorism: biodefense strategy, practice, and Science, vol. 11, suplement 1, 2013.

MANNING, L.; SOON, J. M. Food Safety, Food Fraud, and Food defense: A Fast Evolving Literature. Journal of Food Science, nº 81, pp. 823-834, 2016.

NGANJE, Willian, et. ali. Food safety and defense risks in U.S. - Mexico produce trade. Choices Magazine. 2nd quarter 2009, Number 24 (2), 2009.

PRAIA, Elisabete F. S. S. Avaliação da implementação de requisitos de food defense em unidades alimentares. Dissertação de mestrado. Universidade de Lisboa, 2017.

SAATHOFF, Scott M. Food defense planning and implementation in a food ingredientes manufacturing facility. Master degree, Kansas State University, USA, 2011.

SEVERINO, Paula Rita de Sousa. Food defense e sua relação com as normas IFS V6, BRC V7 e FSSC 22000. Dissertação de mestrado. Universidade de Lisboa, 2016.

SPINK, J., MOYER, D.C. Defining the public health threat of food fraud. Journal of Food Science. $N^{o} 76,2011$, pp. 157-163.

SPINK, J., MOYER, D.C. Understanding and combating food fraud. Food Technology Magazine, vol. 67, $\mathrm{n}^{\circ}$ 1, 2013, pp. 30-35.

UNITED STATES DEPARTMENT OF HEALTH \& HUMAN SERVICES \& UNITED STATES FOOD AND DRUG ADMINISTRATION (HHS \& FDA). Food Safety and Security: Operational Risk Management Systems Approach. Washington, Estados Unidos: FDA, 2001.

UNITED STATES DEPARTMENT OF HEALTH \& HUMAN SERVICES \& UNITED STATES FOOD AND DRUG ADMINISTRATION (HHS \& FDA). CARVER + Shock Primer. Na overview of the Carver Plus Shock method for food sector vulnerability assessments. Washington: Estados Unidos: FDA, 2014. Disponível em: https://www.fda.gov/food/fooddefense/fooddefenseprograms/ucm376791.htm. Acesso em: 15 de junho de 2018.

UNITED STATES DEPARTMENT OF HEALTH \& HUMAN SERVICES \& UNITED STATES FOOD AND DRUG ADMINISTRATION (HHS \& FDA). Food defense awareness. Washington, Estados Unidos: FDA, 2015a. Disponível em: https://www.accessdata.fda.gov/scripts/fdtraining/course_01/module_01/lesson_03/FD01_01 03 060.cfm. Acesso em: 13 de junho de 2018.

UNITED STATES DEPARTMENT OF HEALTH \& HUMAN SERVICES \& UNITED STATES FOOD AND DRUG ADMINISTRATION (HHS \& FDA).Training course for food security. Washington, Estados Unidos: FDA, 2015b. Disponível em: http://www.fda.gov/downloads/Training/ForStateLocalTribalRegulators/UCM218900.pdf. 
UNITED STATES DEPARTMENT OF HEALTH \& HUMAN SERVICES \& UNITED STATES FOOD AND DRUG ADMINISTRATION (HHS \& FDA).FSMA final rule for mitigation strategies to protect food against intentional adulteration. Washington, Estados Unidos: FD, 2016.

WORLD HEALTH ORGANIZATION (WHO). Terrorist threats to food: guidance for establishing and strengthening prevention and response systems. World Health Organization. Geneva, Switzerland, 2002.

WORLD HEALTH ORGANIZATION (WHO). Food defense. World Health Organization. Geneva, Switzerland, 2002. Disponível em: http://www.who.int/trade/glossary/story028/en/. Acesso em: 18 de junho de 2018.

YADAV, V.; SHARMA, A. Free software for food industries to ensure food safety: CARVER + Shock. Comprehensive reviews for food Science and food safety, vol. 10, 2016, pp. 109117. 